\title{
An Environmental Hedging Point Policy to control production rate and emissions in unreliable manufacturing systems
}

\author{
A. Ben-Salem ${ }^{\text {a }}$, A. Gharbi ${ }^{\text {a* }}$, A. Hajji ${ }^{\text {b }}$ \\ a Automated Production Engineering Department, École de technologie supérieure, \\ Production System Design and Control Laboratory, University of Québec, 1100 Notre \\ Dame Street West, Montreal, QC, Canada H3C $1 \mathrm{~K} 3$ \\ b Department of Operations and Decision Systems \& CIRRELT. Laval University. \\ Pavillon Palasis-Prince 2325, rue de la Terrasse Québec (Québec) G1V $0 A 6$
}

\begin{abstract}
$\underline{\text { Abstract: }}$
This paper proposes a new hedging point policy which integrates environmental concerns into the optimal control of unreliable manufacturing systems. The considered system is composed of a production facility subjects to random failures and producing a product family intended for a given market with stable demand. The manufacturing facility's operations cause harmful emissions to the environment, and may incur sanctions in the form of an environmental tax imposed by the relevant authorities. Given the significant compromise that must take place between inventory, backlog and taxes costs, the main objective of this paper is to propose a feedback adaptive control policy which provides a better control of the production rate and the emissions generated. Under the hedging point policies (HPP) category, a new structure called the Environmental Hedging Point Policy (EHPP) is proposed. To illustrate the effectiveness of the proposal, an experimental approach based on simulation modelling, variance analysis and response surface methodology (RSM) is applied. The results show a significant gain in terms of incurred costs compared to those incurred when the system is governed by a classical HPP. An improved version of EHPP is also proposed for systems with high emission rates. Several sensitivity analyses are conducted to illustrate the robustness and effectiveness of the proposed policies.
\end{abstract}

Keywords: Unreliable manufacturing systems, hedging point, emission, production control, simulation, RSM. 


\section{Introduction}

During the past 20 years, the social and economic pressures have led manufacturing firms to pay more attention to the environmental consequences of the products and services they offer and to the processes they deploy (Brandenburg et al. 2014). This reality has brought a new dimension, namely, environmental performance, to the efficiency and traditional performance measures of a business. Therefore, operational strategies must be able to adapt to market changing conditions, react to unforeseen events, and solve such difficulties by collaborating, even while integrating environmental concerns.

From an operational point of view, industrial facilities are facing a lack of strategies developed to meet waste and toxic emissions standards and requirements while maintaining high economic efficiency (Bonney and Jaber, 2011). This lack of strategies is accentuated by the highly dynamic and complex context that governs manufacturing systems. There is obviously a significant gap when it comes to strategies or standards independently addressing both economic and environmental problems.

In the scientific literature, when environmental aspects are ignored, most efforts tend to be focused on monitoring and improving management processes giving rise to several theories. In a dynamic stochastic context, optimal control theory is one of the most contributory in the development of operational manufacturing strategies.

In the context of manufacturing system, feedback control policies are among the most effective strategies in a stochastic dynamic environment. One of the most cited and employed class of strategy in manufacturing systems facing random events (e.g., breakdowns, random demand, etc.), is the Hedging Point Policy (HPP) (Kenne and Gharbi, 2000). In its simplest form, for an M1P1 (one unreliable machine (M) producing one product type (P)), aiming to minimize a long-term discounted cost, HPP acts as a feedback strategy to control the production rate as a function of the state of the system, and calls for a safety stock (threshold) to be built during excess capacity periods in order to be able to meet demand during failure periods. Based on the concept of HPP, many others applied the same formalism to extend this control policy to other manufacturing contexts. Hajji et al. (2011) and Gharbi et al. (2011) developed the Multiple HPP (MHPP) for multiple state systems. Berthaut et al. (2011), Rezg et al. (2005) and Ayed et al. (2012), among others, considered a joint implementation of a corrective and 
preventive maintenance strategy and production rate control. For systems producing multiple part types, Bai and Elhafsi (1997) developed Hedging Corridor Policy (HCP), adapted to this context. Based on this work, Gharbi et al. (2006) extend the problem to a multiple-machine context. Hajji et al. (2009) considered joint replenishment and production control in a two-stage stochastic manufacturing system and developed a statedependent HPP including feedbacks on the raw material and finished products inventories. Recently, process and product quality considerations have been integrated into production planning (Radhoui et al., 2009; Bouslah et al., 2013).

All the aforementioned works - in addition to many others - have considered important aspects of manufacturing system control. However, the integration of the environmental dimension when controlling manufacturing systems in a dynamic stochastic context remains an open problem, and needs to be addressed. In fact, this need is due mainly to the reality faced by the industrial sector, which must combine its economic efficiency goals with environmental standards requirements.

In the literature, few authors address production control activities jointly with environmental aspects at the operational decision level. Some such contributions, cited below, are clearly responding to an urgent need highlighted by a significant increase in environmental legislation and in waste disposal costs (Porter and Linde, 1995). Papers that address the environmental aspect jointly with production planning decisions mainly consider two different approaches, namely, RCA and VA.

Regulatory control approach (RCA): This is a well-known approach used by authorities worldwide to establish standards and rules for controlling and reducing toxic emissions, pollutants and wastes (Chen and Monahan, 2010). Regulatory instruments allow necessary interventions through higher standards and/or taxation to reduce environmental risks (Lee and Yik, 2004).

Bonney and Jaber (2011) were among the first to integrate the environmental dimension into inventory management models. They proposed an extension of the lot sizing model called the "Environ-EQQ". In addition to transportation costs, this model integrates emissions and waste costs. Jaber et al. (2013) addressed the problem within the European Union Emissions Trading System (EU-ETS). They studied and proposed several schemas 
for emission quota exchange. The principal objective of their work was to find the best production plan that minimizes the total, environmental and supply chain incurred costs.

Voluntary approaches (VA): Also known as self-regulation, these approaches are mainly inspired from international EMS (Environmental Management System) standards (Simonet, 2003). These voluntary approaches have become widely known and used as tools for improving efficiency, reducing emissions (Brouhle et al., 2009) and allowing industries to self-regulate (Marcus and Willig, 1997).

According to Chen and Monahan (2010), regulatory and voluntary approaches lead to two distinct processes of production planning and inventory management. In a regulatory context, the company is required to meet standards that most often constitute a capacity constraint for it. However, voluntary control is an internal choice in which environmental objectives are set by the decision support system. Generally, voluntary approaches provide more flexibility for manufacturing systems in terms of capacity management.

In the light of this new reality and the increasing needs, the main contribution of this paper is to provide decision makers with manufacturing strategies that incorporate both economic and environmental visions. Thus, a stochastic optimal control problem of a manufacturing facility emitting pollutants is considered. The objective is to develop a control policy falling under the class of HPPs that provide the best way to manage incurred costs and generated emissions within an environmental control approach.

The paper is organized as follows. Section 2 presents a description of the system and the stochastic optimal production control problem considered. In the same section, a description of the system's dynamic evolution and the structure of the proposed control policy are presented. Section 3 introduces the proposed resolution approach and presents the simulation model. The other steps are applied in section 4 to solve the problem under a linear relationship between the emission and production rates. Section 5 extends the problem analysis for a case of an exponential form of emission. Section 6 concludes the paper.

\section{Problem statement}

\subsection{Notations}

The following notations are used: 


\begin{tabular}{|c|c|}
\hline$x(t)$ & Finished product inventory/backlog level \\
\hline$u(t)$ & Production rate \\
\hline$e(t)$ & Emission level \\
\hline$U_{\max }$ & Maximum production rate \\
\hline$d$ & Finished product demand rate \\
\hline $\boldsymbol{L}$ & Standard permitted limit of emission \\
\hline $\boldsymbol{Y}$ & Voluntary limit of emission \\
\hline $\boldsymbol{\theta}$ & Emission index \\
\hline $\boldsymbol{P}_{\text {er }}$ & Length of emission control period \\
\hline $\boldsymbol{N}$ & Number of periods in the planning horizon \\
\hline$\rho$ & Discount rate \\
\hline$\alpha(t)$ & Discrete variable describing the manufacturing system state \\
\hline MTTF & Mean Time To Failure \\
\hline MTTR & Mean Time To Repair \\
\hline$C^{+}$ & Finished product holding cost/Unit/Time unit (TU) \\
\hline$C$ & Finished product backlog cost/Unit/TU \\
\hline$C^{e}$ & Penalty cost for emissions/Unit \\
\hline$Z i$ & Finished product Hedging level, $\mathrm{i}=1,2$. \\
\hline
\end{tabular}

\subsection{Problem description}

The manufacturing system under study (Figure 1) consists of an unreliable production facility producing one product family type to satisfy a constant demand directly from a finished product $(F P)$ stocking area. The manufacturing system is unreliable, which causes periods of unavailability (failures) requiring repairs to restore the system. Failure events and repairs duration are assumed to evolve according to a stochastic process. Due to the unavailability periods of the system, unsatisfied demands are backlogged, with a penalty cost. The manufacturing facility's operations cause harmful emissions to the 
environment and may incur sanctions in the form of an environmental tax imposed by the relevant authorities. We assume that there is no emission caused by the products in work in process (WIP). Given the significant compromise that must take place between inventory, backlog and emissions penalty costs, the main objective of this paper is to propose a feedback adaptive policy which provides a better control of the production rate and the emissions generated.

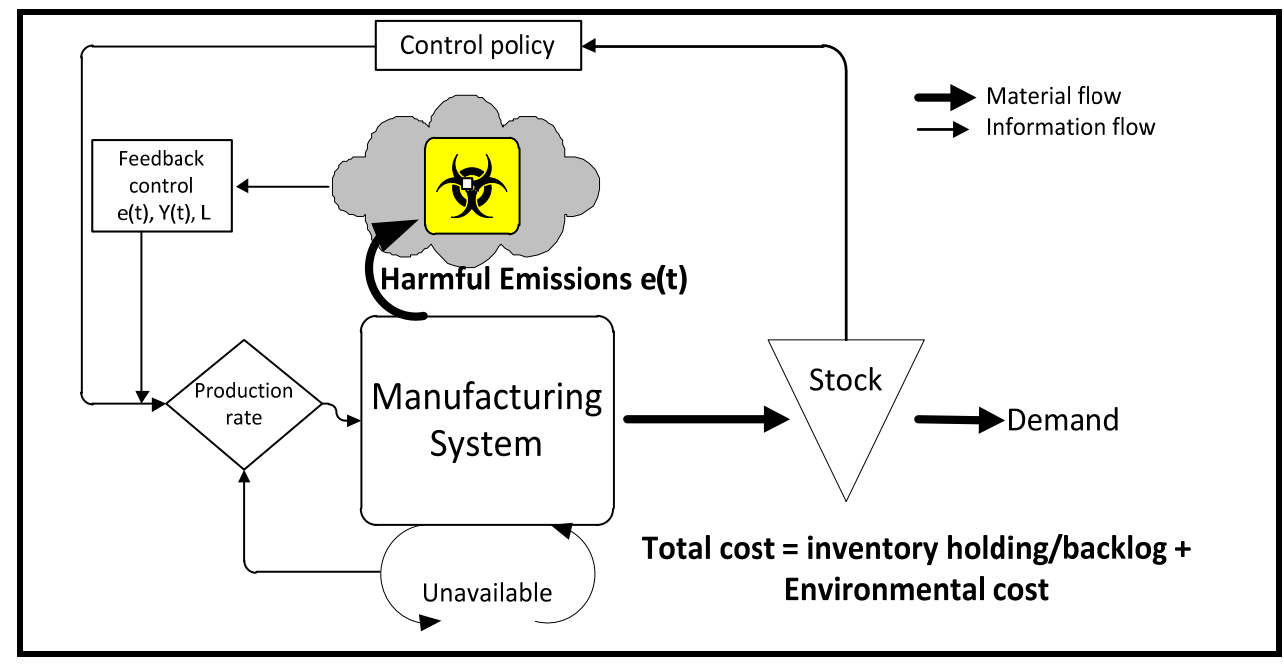

Figure 1: System under study.

For the considered manufacturing system, $x(t)$ and $u(t)$ denote the inventory/backlog level of $F P$ and the production rate of the system.

For any specific time $t$, the state of the system has two components: a continuous component denoted $x(t)$ describing the cumulative surplus level, and a discrete component denoted $\alpha(t)$ describing the manufacturing system state. $\alpha(t)$ is a continuoustime discrete space stochastic process taking value: 0 if the system is under repair and 1 if it is operational. Hence, the dynamic behaviour of the system can be modelled by the state variables $(x(t), \alpha(t))$, where $x(t) \in R, \alpha(t) \in M=\{0,1\}$.

The dynamic behaviour of the FP surplus is given by the following differential equation:

$$
\dot{x}(t)=u(t)-d, x(0)=x_{0}
$$

where $x_{0}$ denotes the initial surplus level.

The production rate at any given time must satisfy the capacity constraint of the system given by equation (2).

$$
0 \leq u(t) \leq U_{\max }
$$


When processing parts at a fixed rate $u(t)$, the system is constrained to emit a quantity of harmful pollutants for each part produced. Let $\theta$ be the emission index expressed as the quantity of pollutants per unit produced. The dynamic behaviour of the quantity of emissions is given by equation (3):

$$
\dot{e}(t)=u(t) \times \theta, \quad t \in\left[t_{i}, t_{i+1}\left[, e\left(t_{i}\right)=0, i=0, . ., N,\right.\right.
$$

Where $N$ denotes the number of periods in the planning horizon.

Following the aforementioned Regulatory control approach (RCA), the manufacturing facility under study must comply with the standards and rules that stipulate that in each reference period $i$, if the quantity of emissions exceeds a standard level $\mathrm{L}$ fixed by the relevant authorities, the excess quantity is penalized with an environmental $\operatorname{cost} \mathrm{C}^{\mathrm{e}}$ (Jaber et al. 2013). At the end of the reference period, the emission counter is reset to zero.

For a better comprehension of the emission indices $\theta$, we refer the reader to the work of Chen and Manahan (2010).

The decision variable of the control policy we are seeking is the production rate $u(t)$ which can have tree values; $u(t)=0$ if the system is shut down; $u(t)=d$ or $u(t)=U_{\max }$ if the system is available.

The decision made by the manager is strongly conditioned by the involved costs defined in the following equations.

At time $t$, we calculate the inventory and backlog cost according to the inventory level $x(t)$. The instantaneous cost function $g($.$) is given by the following equation:$

$$
g(x(t))=C^{+} x^{+}+C^{-} x^{-}
$$

Where $x^{+}=\max (0, x), x^{-}=\max (-x, 0), C^{-}$is the $F P$ backlog cost and $C^{+}$is the FP inventory cost.

The penalty emission cost at the end of reference periods $i$ is given by the following equation:

$$
E C\left(t_{i}\right)=C^{e} \times \max \left(0, e\left(t_{i}\right)-L\right), i=0, . ., N
$$

Using the equations (4) and (5), the average total cost $J($.) per unit of time can be defined by the following equation: 


$$
J(x, e, \alpha)=\int_{0}^{\infty} e^{-\rho t} g(x(t)) d t+\frac{\sum_{i=1}^{N} C^{e} \times \max \left(0, e\left(t_{i}\right)-L\right)}{N \times P_{e r}}
$$

The production planning problem considered here is to find an admissible decision or control policy $u($.) that minimizes $J($.), given by (6) subject to equations (1) to (3). Such a feedback control policy, as illustrated in Figure 1, determines the production rates as a function of $x(t), \alpha(t)$ and $e(t)$.

In the following section, an extended version of HPP is proposed to control such a system.

\subsection{Environmental Hedging Point Policy (EHPP)}

Recall that the classical HPP doesn't consider the emission costs. The control of the system under study will confront the manager with the need for an important trade-off between backlog costs, if the produced quantity is not sufficient, and the inventory and emission cost, if the produced quantity is very significant. The simplest way to tackle the problem will be to never exceed the permitted limit L. Unfortunately, in an unreliable manufacturing context, the manager, in order to remain competitive in the market, cannot afford to permanently limit production and ignore the possibility of an occasional emissions overflow. In the light of this reality, starting from the classic HPP, which requires production to be carried out at the maximum rate to reach a hedging level $Z_{1}$, an additional feedback information from the emission level $e(t)$ is needed in order to improve production planning decisions. In this context, the manager should have an adapted emission control level beyond which he can decide to stop production if the emission cost rises. This decision cannot be taken independently of the inventory level, and thus a coupled feedback control should be considered. This could be inspired from the multiple HPP (MHPP), in which case the manager could decide to stop production if the emission level becomes high and the inventory level is judged sufficient $\left(\geq Z_{2}\right)$, in order to minimize backlog risks. Based on HPP and MHPP, a new control policy called the Environmental Hedging Point Policy (EHPP) is proposed. This policy is a voluntary commitment which consists in setting a specific limit that controls the production rate, based on the inventory and emission levels.

The following equations (7) and (8) summarize the EHPP policy: 


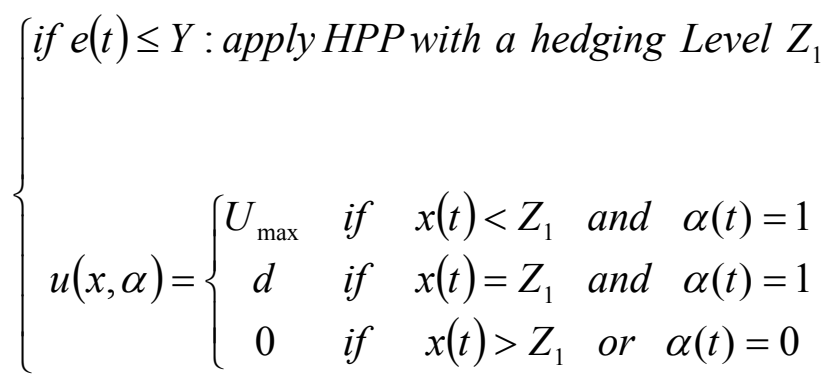

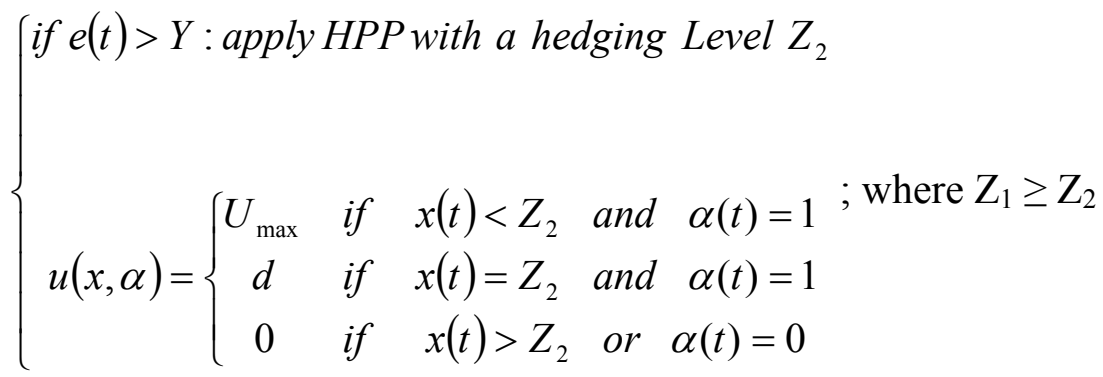

Figure 2, presents a hypothetical evolution of $F P$ inventory level and the way in which EHPP decisions should be taken following equations (7) and (8).

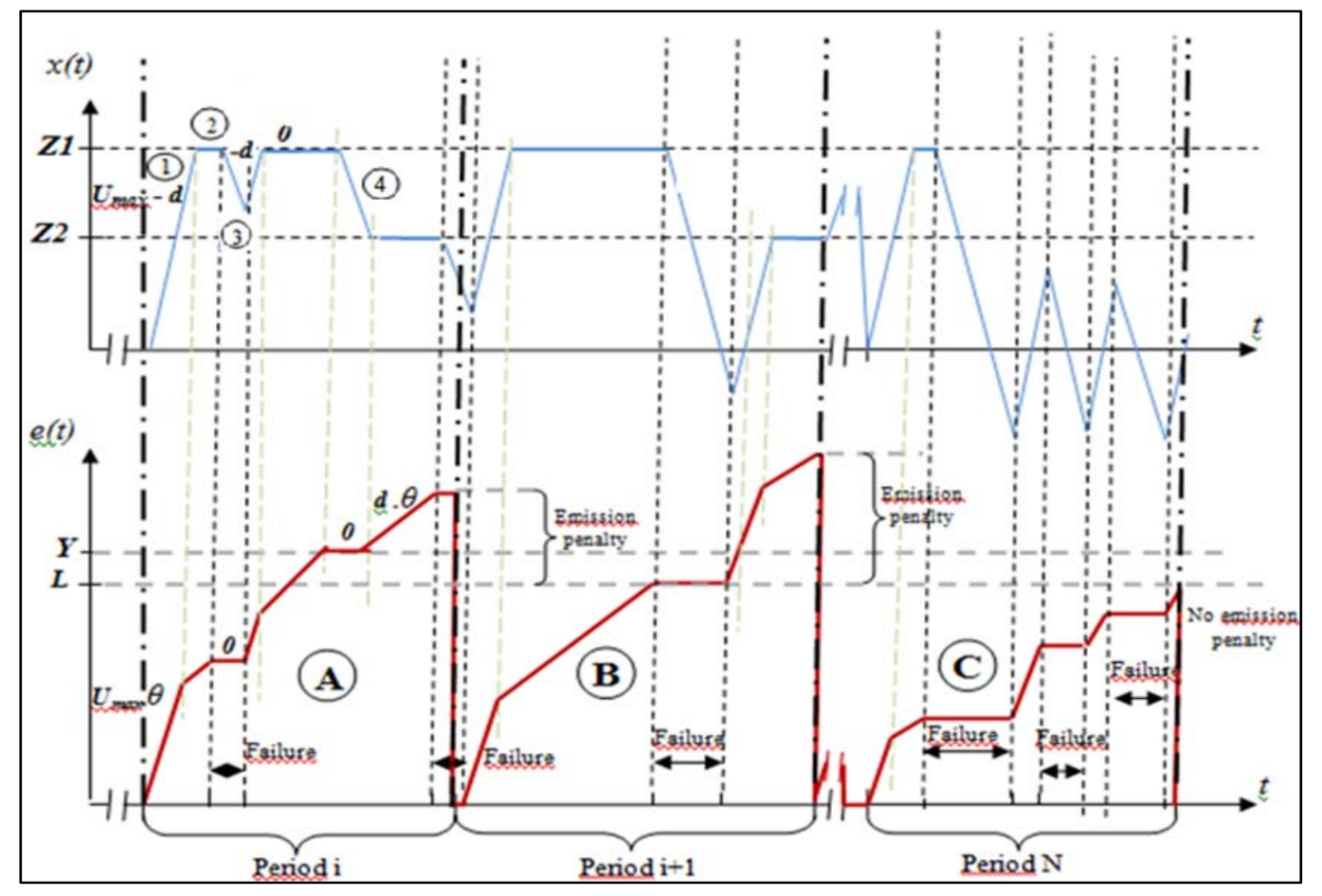

Figure 2: Inventory and emission levels evolution under EHPP

(1): When producing at a maximum rate, the inventory level $x(t)$ rises according to a rate equal to $\left(U_{\max }-d\right)$; the level of production allows demand to be satisfied, and the remaining products are stocked and used to build the hedging level $Z_{1}$. Given that the 
emission rate is synchronized with the production rate, the level of the emission rises according to $U_{\max } \times \theta$.

(2): When $x(t)$ reaches $Z 1$, the production rate is set to the demand rate $d$, and consequently, the emission rate is decreased to $\dot{e}(t)=d \times \theta$.

(3): When a failure event occurs, production processing and emission are stopped $(u(t)=$ 0 ), and consequently, the inventory level decreases according to $(-d)$, while the emission level remains stable.

(A) and (B): During a given reference period, the cumulative quantity of emissions may exceed the standard limit $\mathrm{L}$ set by the relevant authorities. This is the case for periods $\mathrm{i}$ and $i+1$ in Figure 2. In such situations, a penalty must be paid for every excess unit of emission. When the emission level exceeds the voluntary limit $\mathrm{Y}$, the hedging level becomes $Z_{2}$, which is lower than $Z_{1}$ (4). In this case, reaching the security level $Z_{2}$ is enough to reduce production, and consequently, the emissions. At the end of each reference period, the emission counter is reset to zero.

(C): When a failure event occurs, production and emissions are stopped, the inventory level decreases with the possibility of backlogs, as shown for period N. In this case, if the emission level is lower than L, no penalty is imposed.

In comparison with the HPP, EHPP allows the manager the ability to adapt the production policy according to environmental and system constraints. In the following sections, the proposed policy is implemented and several experiments and comparative studies are developed to show its effectiveness.

\section{Resolution approach and simulation model}

3.1. Resolution approach steps

To solve the problem and optimize the policies parameters, an experimental approach integrating simulation, design of experiment (DOE) and RSM is adopted as in Berthaut et al. (2011). The main steps of the experimental resolution approach are:

- $\quad$ Step 1: Establishing of the control policies 
In section 2, the structure of EHPP is presented, analysed and expressed by mathematical equations. Regarding HPP, we refer the reader to Kenne and Gharbi (2000). These policies will govern our simulation models.

- $\quad$ Step 2: Simulation models

Each simulation model (see section 3.2) is designed to reflect the system dynamics governed by one of the control policies considered (HPP and EHPP) in order to compare each one to the other. These policies are used as an input to conduct several experiments, and thus evaluate their costs.

- $\quad$ Step 3: Experimental design and response surface methodology

The experimental design approach defines the number of experiments, the experimental space of the independent variables considered, and the variation extent of each factor. The analysis of variance (ANOVA) is subsequently used to determine the main factors and their interactions which have a significant effect on the cost (dependent variable). Then, the RSM allows us to determine the relationship between the cost and the significant factors. The resulting model is then optimized in order to determine the best combination of factors which minimizes the total cost.

\subsection{Simulation models}

Using the simulation language SIMAN under «ARENA» software, a combined discretecontinuous model is developed with $\mathrm{C}++$ routines. This type of modeling has an advantage in terms of shortening simulation run time (Lavoie et al. (2010)). Figure 3 illustrates the simulation model diagram under the control policy EHPP. Under the control policy HPP the same diagram illustrates the simulation model, but with $Z_{1}=Z_{2}$. 


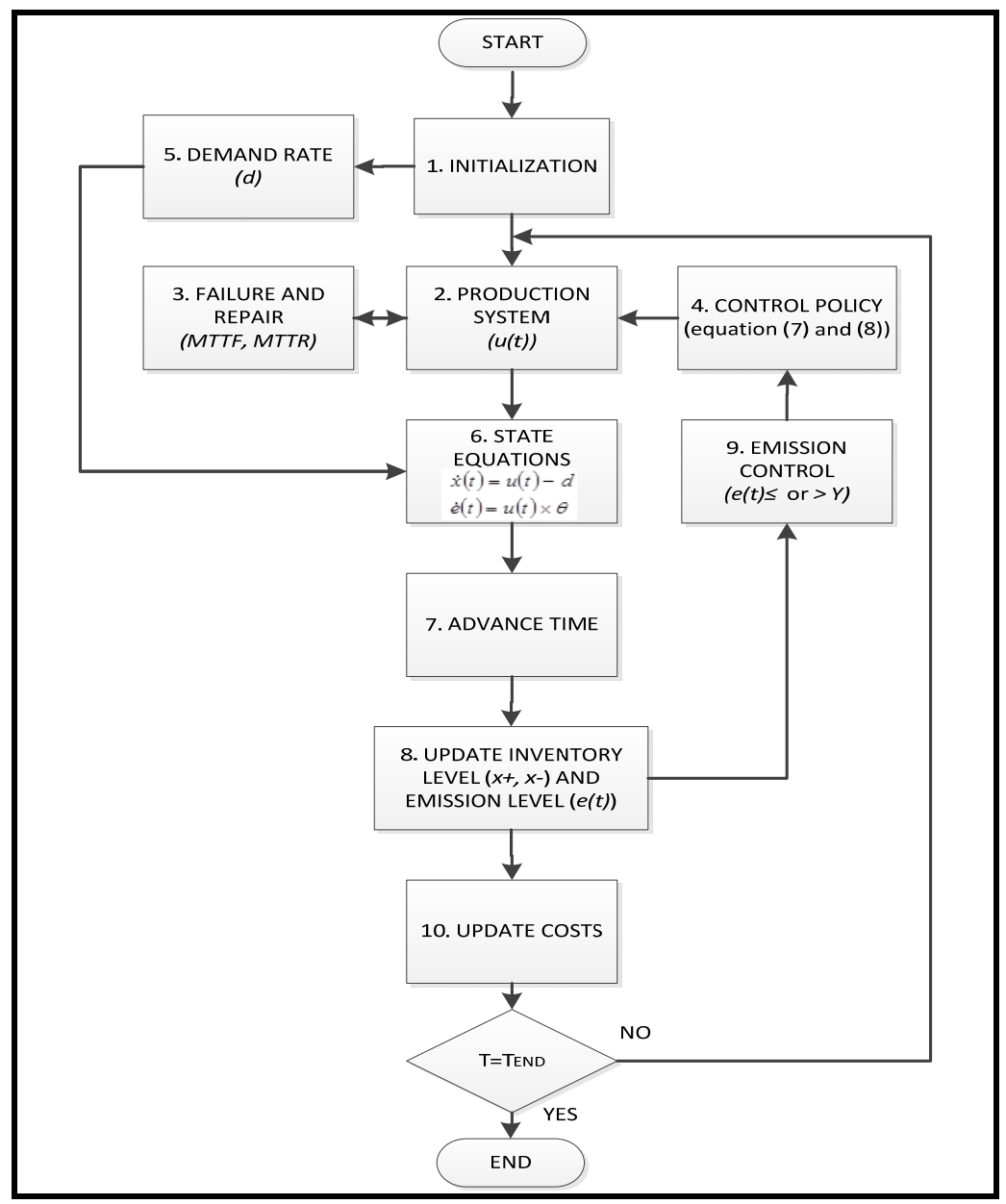

Figure 3: Diagram of the simulation model.

According to Figure 3, after initializing the model with the necessary simulation parameters and inputs $\left(Z, U_{\max }, d\right.$, simulation time ...) (block 1), the production system (block 2), subject to random failures and repairs activities (block 3), allows to produce according to the control policy (block 4) described by equations (7) in order to meet the demand (block 5). The state equations (block 6), which are defined using $\mathrm{C}++$ routines, describe the variation of inventory levels $x(t)$ and the emission levels $e(t)$ according to the equations (1) and (3). The simulation time advance (block 7) and the model updated the level of surplus inventory and the emission level generated (block 8). The control of the emission level, in a reference period i (block 9), allows to check the condition $(e(t)>Y)$. When $e(t)$ exceeds the level $Y$, production control policy (block 4) changes and the system produces according to the equation (8). Finally, a calculation of each cost (inventory, backlog, and emission cost) is executed (block 10). 


\section{Numerical examples: Linear emission rate case study}

This section uses the resolution approach adopted in order to calculate the optimal total cost and optimal values of parameters defining the control policies. It is followed by a sensitivity analysis and a comparison of the HPP and EHPP. Regarding the emissions aspect, we consider a random emission index $\theta$ varying uniformly in a given interval [a,b] as in Chen and Monahan (2010). Table 1 summarizes the considered system parameters for the first numerical example.

Table 1: Values of the system parameters

\begin{tabular}{|c|c|c|c|c|c|c|c|c|c|c|}
\hline Parameters & $d$ & $\mathbf{U}_{\max }$ & MTTF & MTTR & L & $\mathbf{C}^{+}$ & $\mathbf{C}^{-}$ & $\mathbf{C}^{\mathbf{e}}$ & $\mathrm{Per}$ & {$[\mathrm{a}, \mathbf{b}]$} \\
\hline Values & 100 & 130 & $\operatorname{Exp}(7 \mathrm{TU})$ & $\operatorname{Exp}(0.4 \mathrm{TU})$ & 650000 & 1 & 25 & 5 & $5760 \mathrm{TU}$ & {$[0.5,2]$} \\
\hline
\end{tabular}

\subsection{RSM model and optimization}

Regarding HPP, we use a polynomial regression model in order to optimize its unique parameter (Z) (Kenne and Gharbi, 2000). A polynomial regression is performed using the statistical software STATGRAPHICS. The results of this analysis are presented in Table 2.

Table 2: Polynomial regression results for HPP

\begin{tabular}{|c|c|c|c|c|}
\hline Parameter & Estimate & S. Error & T. Statistic & P-Value \\
\hline CONSTANT & 228,676 & 2,35342 & 97,1677 & 0,0000 \\
\hline $\mathbf{Z}$ & $-0,839675$ & 0,0373857 & $-22,4598$ & 0,0000 \\
\hline $\mathbf{Z}^{2}$ & 0,00479738 & 0,000134017 & 35,7969 & 0,0000 \\
\hline
\end{tabular}

The model which respects the convexity property of the cost function is given by equation (9). The results show that the adjusted correlation coefficient $\mathrm{R}^{2}$ adjusted is equal to $98.22 \%$. This means that more than $98 \%$ of the observed variability of the expected total cost is explained by the model (Montgomery, 2005). In the same direction, an analysis of the residual normality and of the homogeneity of variance was also carried out to check the conformity of the models.

$$
\widehat{\operatorname{CoSt}}_{H P P}=228.67-0.839675 \times Z+0.00479738 \times Z^{2}
$$

The minimum total cost is observed at point $\mathrm{Z}^{*}=87.51$, with a value of $\operatorname{Cost}_{\mathrm{HPP}}^{*}=$ 191.93.

On the other hand, for the EHPP policy, we select a full factorial design with 3 factors, at 3 levels each. The full factorial of such a plan is often used because it gives more 
accurate results since each interaction is estimated separately. Five replications were performed for each combination of factors, meaning therefore that a total of $135\left(3^{3} * 5\right)$ simulation experiments were performed. For both simulation models, the simulation length was set to 500.000 units of time. That is long enough to reach the steady state. Regarding the design factors of EHPP, several preliminary simulation experiments were performed to set their levels, as detailed in Table 3 . To ensure the constraints $\left(Z_{2} \leq Z_{1}\right)$, a substitute parameter $r=Z_{2} / Z_{1}$ is introduced, with $0 \leq r \leq 1$.

Table 3: Experimental domain

\begin{tabular}{|c|c|c|c|}
\hline Factor & Low & Medium & High \\
\hline Y & 600000 & 725000 & 850000 \\
\hline Z1 & 35 & 67.5 & 100 \\
\hline r & 0.01 & 0.5 & 0.99 \\
\hline
\end{tabular}

The effects of independent variables $\left(Z_{1}, r, Y\right)$, their interactions, and their quadratic effect on the response variables (cost) were obtained thanks to a multi-factorial ANOVA. The results of this analysis summarized in Table 4 show that all the main factors, their interaction (except $\left(Y \times Z_{1}\right)$ and $\left.\left(Z_{1} \times r\right)\right)$ and their quadratic effects are significant at a $95 \%$ level of significance. The adjusted correlation coefficients ( $R_{\text {adjusted }}^{2}$ ) equal to $97.18 \%$ show that more than $97 \%$ of the variability is explained by the RSM model is given by equation (10).

Table 4 : ANOVA results for EHPP.

\begin{tabular}{|c|c|c|c|c|c|c|}
\hline Source & $\begin{array}{c}\text { Sum of } \\
\text { Squares }\end{array}$ & $\boldsymbol{D f}$ & $\begin{array}{c}\text { Mean } \\
\text { Square }\end{array}$ & F-Ratio & P-Value & Signification \\
\hline $\boldsymbol{A}: \boldsymbol{Y}$ & 2025,92 & 1 & 2025,92 & 167,61 & 0,0000 & $\mathrm{~S}$ \\
\hline $\boldsymbol{B}: \boldsymbol{Z}_{\boldsymbol{1}}$ & 41332,0 & 1 & 41332,0 & 3419,43 & 0,0000 & $\mathrm{~S}$ \\
\hline $\boldsymbol{C}: \boldsymbol{r}$ & 1234,46 & 1 & 1234,46 & 102,13 & 0,0000 & $\mathrm{~S}$ \\
\hline $\boldsymbol{A} \boldsymbol{A}$ & 843,214 & 1 & 843,214 & 69,76 & 0,0000 & $\mathrm{~S}$ \\
\hline $\boldsymbol{A} \boldsymbol{B}$ & 17,405 & 1 & 17,405 & 1,44 & 0,2324 & $\mathrm{~N} . \mathrm{S}$ \\
\hline $\boldsymbol{A C}$ & 2205,76 & 1 & 2205,76 & 182,49 & 0,0000 & $\mathrm{~S}$ \\
\hline $\boldsymbol{B} \boldsymbol{B}$ & 8812,29 & 1 & 8812,29 & 729,05 & 0,0000 & $\mathrm{~S}$ \\
\hline $\boldsymbol{B C}$ & 25,0528 & 1 & 25,0528 & 2,07 & 0,1525 & $\mathrm{~N} . \mathrm{S}$ \\
\hline $\boldsymbol{C C}$ & 174,661 & 1 & 174,661 & 14,45 & 0,0002 & $\mathrm{~S}$ \\
\hline Total error & 1510,92 & 125 & 12,0874 & & & \\
\hline Total (corr.) & 58181,6 & 134 & & & & \\
\hline
\end{tabular}




$$
\begin{aligned}
& \widehat{\operatorname{CoSt}}_{E H P P}=557.474-0.00057944 \times Y-2.84992 \times Z 1-89.3766 \times r+ \\
& 3.391810^{-10} \times Y^{2}+0.0000989915 \times Y \times r+0.0162262 \times Z 1^{2}+10.0495 \times \\
& r^{2}
\end{aligned}
$$

The projections of the cost response surfaces on two-dimensional planes are presented in Figure 4.
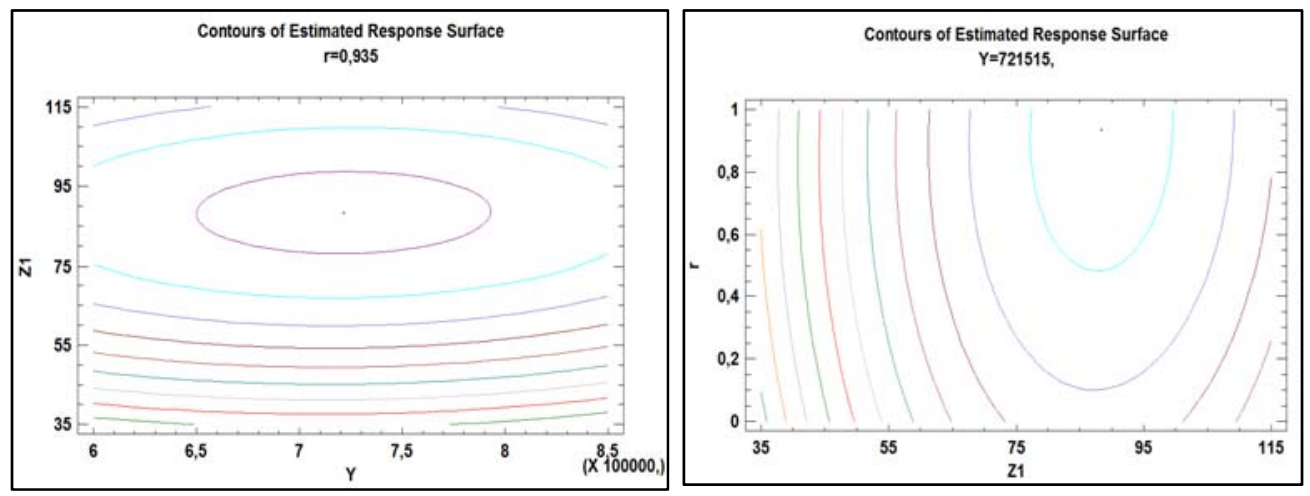

Figure 4: Response surfaces contour plots for the total cost

The minimum total cost obtained is equal to $\operatorname{Cost}_{\mathrm{EHPP}}^{*}=182.54$ which corresponds to the optimal control parameters, $\mathrm{Z} 1 *=88.35, \mathrm{r}^{*}=0.935\left(\mathrm{Z} 2^{*}=82.61\right)$ and $\mathrm{Y}^{*}=721515$.

The optimization results obtained for HPP and EHPP show that for selected system parameters (Table 1), EHPP is more advantageous in terms of cost than the classical HPP, with a $4.89 \%$ reduction. This improvement is mainly due to the ability of EHPP to better control the production rate and the generated emissions such as to arrive to a better compromise of costs. To cross-check the validity of the models represented by equations (9) and (10), we confirm that the optimal cost for each control policy falls within the confidence interval at the 95\% level; [181.85, 183.4] and [191.31, 193.37] for EHPP and HPP, respectively.

\subsection{Sensitivity analysis: $\left(\mathrm{C}^{+}, \mathrm{C}^{-}\right.$and $\left.\mathrm{C}^{\mathrm{e}}\right)$}

In order to confirm the robustness of the resolution approach employed as well as the advantage of EHPP compared to HPP in different scenarios, an extensive sensitivity analysis was conducted. The results of this analysis are presented in the next subsections.

Figure 5 shows the variation of the three design parameters of EHPP when $\mathrm{C}^{+}$varies from 0.8 to 1.2 (respectively $\mathrm{C}^{-}$varies from 24 to 28 ). When $\mathrm{C}^{+}$increases (respectively $\mathrm{C}$ decreases), the system reacts by decreasing the values of the optimal hedging levels. 
Thus, $Z_{1}$ and $Z_{2}$ decrease. When $C^{+}$decreases (respectively $C$ - increases), the opposite occurs. The same figure also shows the effects of the "penalty cost for emissions" $\mathrm{C}^{\mathrm{e}}$ on the optimal hedging levels. Thus, when $C^{\mathrm{e}}$ decreases, $Z_{2}$ tends to approach $Z_{1}$. For low values of $\mathrm{C}^{+}$(respectively for high values of $\mathrm{C}^{-}$), this trend is accelerated and $\mathrm{Z}_{2}$ tends to get too close to $Z_{1}$. At the limit, when $C^{\mathrm{e}}$ is negligible, EHPP becomes equal to HPP, given that $Z_{2}$ tends to be equal to $Z_{1}$.

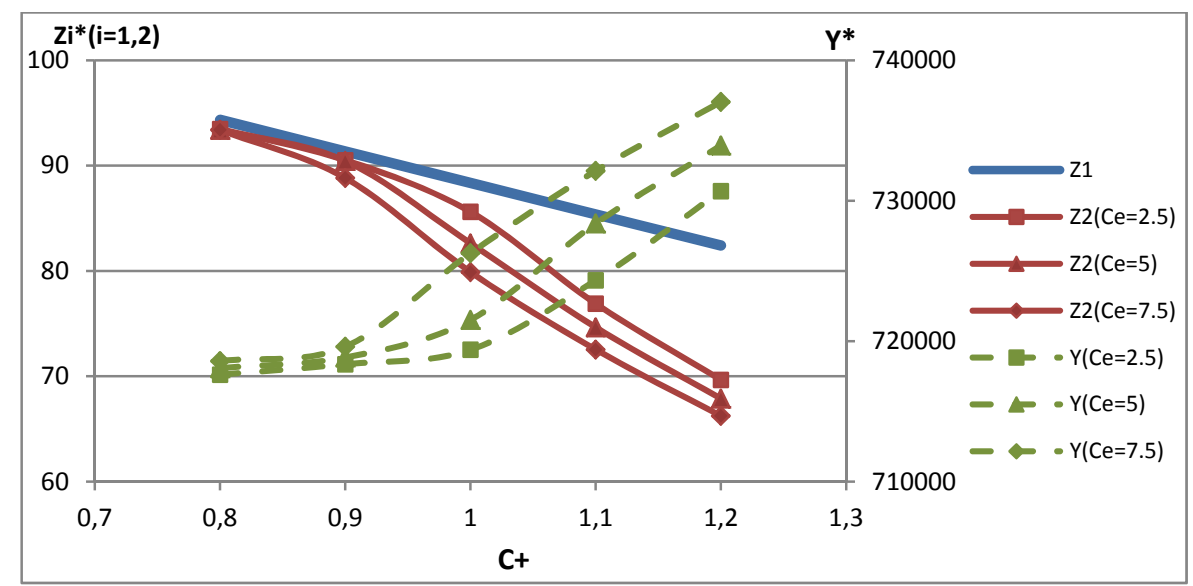

a. $\left(\mathrm{C}^{+}\right)$

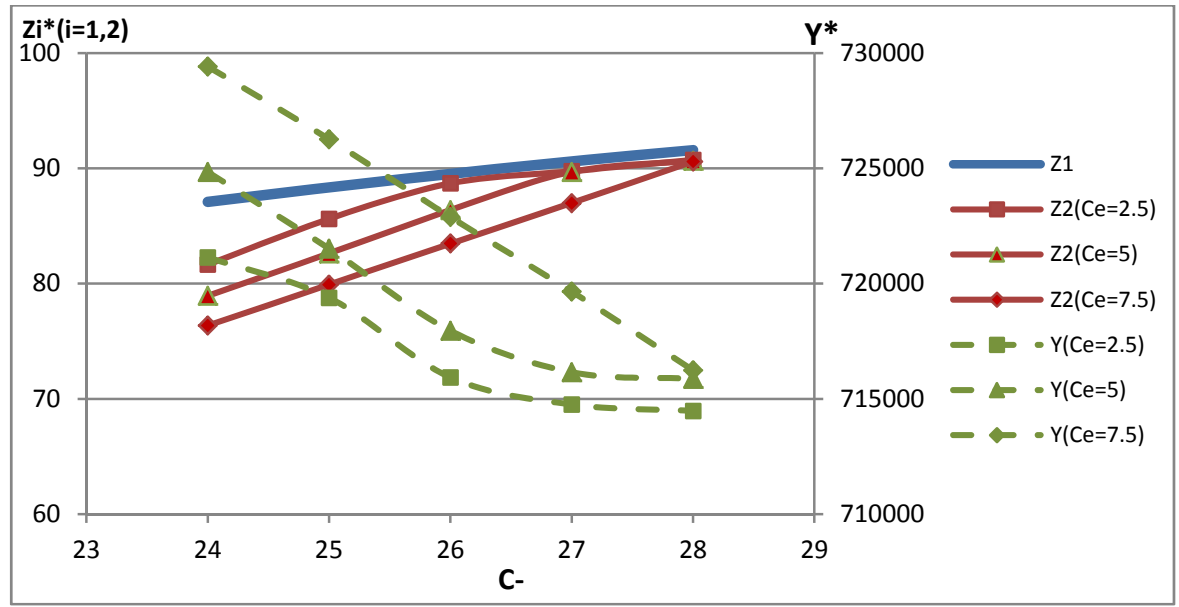

b. $\left(\mathrm{C}^{-}\right)$

Figure 5: Variation of $\mathrm{Zi}^{*}(\mathrm{i}=1,2)$ and $\mathrm{Y}^{*}$ when varying $\mathrm{C}^{+}, \mathrm{C}^{-}(\mathrm{EHPP})$

With respect to the variation of $\mathrm{Y}$ in Figure 5, we can observe that it is the opposite of that of $Z_{1}$ and $Z_{2}$. In fact, when the costs $\left(C^{+}\right.$or $\left.C^{-}\right)$lead to high values of $Z_{1}$ and $Z_{2}$, it means that the system will produce more at the maximum rate, a situation which leads to more emissions. To limit this increase, the system reacts by reducing the values of $\mathrm{Y}$, leading to an earlier stoppage of production. This reaction is also a function of the 
"penalty cost for emissions" $\mathrm{C}^{\mathrm{e}}$. From Figure 5, we can also observe that when $\mathrm{C}^{\mathrm{e}}$ increases, $Z_{2}$ decreases, leading to a higher risk of backlogs. Thus, higher values of $\mathrm{Y}$ are required to maintain the inventory level longer at $\mathrm{Z}_{1}$.

Figure 6 show that when varying $C^{\mathrm{e}}$ no effect is observed on $Z_{1}$. This makes sense since the hedging level $\mathrm{Z}_{1}$ mainly governs the production rate, and is mostly sensitive to $\mathrm{C}^{+}$and $\mathrm{C}^{-}$. However, the variation of $\mathrm{C}^{\mathrm{e}}$ mainly affects the hedging level $\mathrm{Z}_{2}$. As explained in the last paragraph, when $\mathrm{C}^{\mathrm{e}}$ increases, the system reacts by limiting emissions through a reduction of the level of $\mathrm{Y}$ from which production is stopped. When $\mathrm{C}^{\mathrm{e}}$ decreases, the gap between the two hedging levels is reduced, leading to the classical HPP for negligible $\mathrm{C}^{\mathrm{e}}$.

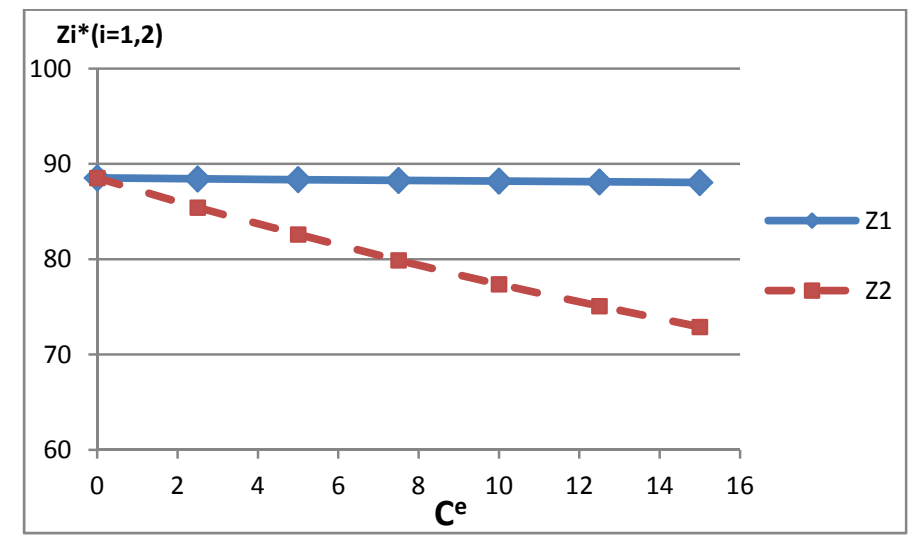

Figure 6: Variation of $\mathrm{Zi}(\mathrm{i}=1,2)$ when varying $\mathrm{C}^{\mathrm{e}}$ (EHPP)

The same sensitivity analysis was conducted for the HPP. In this context, the variations of the design parameter $Z$ when varying the cost parameters are the same as $Z_{1}$ for EHPP. It is also interesting to note that for all cases, we observed an average gain equal to $4 \%$ for the total cost under EHPP compared to that under HPP.

\subsection{Improved HPP and EHPP: Production rate optimization}

In the literature of optimal control (see Section 1), few works consider the maximum production rate as a design parameter to be optimized as in Sana and Chaudhuri (2010) and Giri and Dohi (2005). Whether or not the maximum production rate is considered as a design parameter is directly dependent on whether or not the production rate is penalized in the objective function. In the case of HPP, only inventory and backlog costs are penalized. It is always in the interest of the system to produce at high rates $U_{\max }$ in 
order to quickly reach the security hedging level. Recall that in our case, when emission limit is exceeded, an emission tax is added. This cost indirectly penalizes the production rate, given the relationship (3). Thus, we argue that the optimization of the production rate within HPP and the proposed EHPP could improve performance.

In the literature, Jaber et al. (2013) were among the first who considered the production rate as a design parameter to optimize in a context including environmental aspects. However, the problem considered in Jaber et al. (2013) did not take into account the operational activities at the manufacturing level. In this section, we discuss the situation in which the production rate is considered as a decision variable, in addition to the decision variables $\left(\mathrm{Z}_{1}, \mathrm{Z}_{2}\right.$ and $\left.\mathrm{Y}\right)$ for $\mathrm{EHPP}$, and $\mathrm{Z}$ for HPP.

Considering the same system parameters (see Table 1), the maximum production rate should be set to meet the feasibility constraint given by formula (11). Thus, the maximum production rate should be at least equal to 105.72, given that the system is available $94.59 \%$ of the time, and the demand rate is equal to 100 .

$$
U_{\max } \times \frac{M T T F}{M T T F+M T T R} \geq d
$$

The experimental domain of the decision variable $U_{\max }$ is given in Table 5. The improved proposed policies, named HPPU and EHPPU, are represented by the same equations (7) and (8), but $U_{\max }$ should be optimized together with $\left(\mathrm{Z}_{1}, \mathrm{Z}_{2}\right.$ and $\left.\mathrm{Y}\right)$.

Table 5: Experimental domain of $U_{\max } *$

\begin{tabular}{|c|c|c|c|}
\hline Factor & Low & Medium & High \\
\hline $\mathrm{U}_{\max }$ & 110 & 120 & 130 \\
\hline
\end{tabular}

Following the same approach (Section 4.1), and for the same set of system parameters (Table1), the results obtained showed that the RSM models have very good adjusted correlation coefficients $\mathrm{R}_{\text {adjusted, equal to }}^{2} 98.88 \%$ for HPPU and $98.57 \%$ for EHPPU. These models are given by equations (12) and (13) for EHPPU and HPPU, respectively. 


$$
\begin{aligned}
& \widehat{\operatorname{CoSt}}_{\text {EHPPU }}=32569.9-0.00025806 \times Y-33.1701 \times Z 1-238.334 \times r-484.796 \times \\
& U_{\max }+0.0000418142 \times Y \times r+0.00000124433 \times Y \times U_{\max }+0.0198931 \times Z 1^{2}+ \\
& 0.230125 \times Z 1 \times U_{\max }+1.4625 \times U_{\max } \times r+1.8241 \times U_{\max }^{2} \\
& \widehat{\operatorname{Cost}}_{H P P U}=16165.9-26.0288 \times Z 1-235.284 \times U_{\max }+0.0191186 \times Z 1^{2}+ \\
& 0.17877 \times Z 1 \times U_{\text {max }}+0.868991 \times U_{\text {max }}^{2}
\end{aligned}
$$

Table 6 gives the optimal design parameters and costs for HPPU and EHPPU. The results obtained clearly show a marked improvement. The production rate optimization led to an average cost reduction equal to $18.9 \%$ and $21.9 \%$ compared to HPP and EHPP, respectively (see Section 4.1). In conclusion, the EHPPU policy is the best policy to consider in our case.

Table 6: Optimization results for HPPU and EHPPU

\begin{tabular}{|l|c|c|c|c|c|c|}
\hline Model & $\mathrm{Z}_{1}$ & $\mathrm{Z}_{2}$ & $\mathrm{Y}$ & $\mathrm{U}_{\max }$ & Total cost * & Confidence interval (95\%) \\
\hline HPPU & 92.06 & - & - & 125.91 & 155.65 & {$[155.22 ; 156.42]$} \\
\hline EHPPU & 110.18 & 91.9 & 1061520 & 125.24 & 142.57 & {$[141.86 ; 143.07]$} \\
\hline
\end{tabular}

\subsection{Sensitivity analysis}

In Section 4.2, we studied the effect of the holding and backlog costs on the critical thresholds (Z1 and Z2). In the same vein, from Figure $7 \mathrm{a}$ and $7 \mathrm{~b}$, we note that when the parameter $\mathrm{C}^{+}$increases (respectively $\mathrm{C}^{-}$decreases), $\mathrm{Z} 1$ and $\mathrm{Z} 2$ decrease. Thus, the maximum production rate increases because a high production rate allows to quickly build the comfortable thresholds. Regarding the emissions, the increase in Z1 and Z2 increase the emissions (see Section 4.2), and consequently, the production rate decreases in order to limit this increase.

Regarding the penalty cost, Figure 7c shows that the optimal production rate decreases when $\mathrm{C}^{\mathrm{e}}$ increases, leading to a decrease in the emission rate. This result, together with the explanation of Figures $7 \mathrm{a}$ and $7 \mathrm{~b}$, can be supported by the fact that the system must adjust its maximum production rate to avoid exceeding the permitted emission level. 


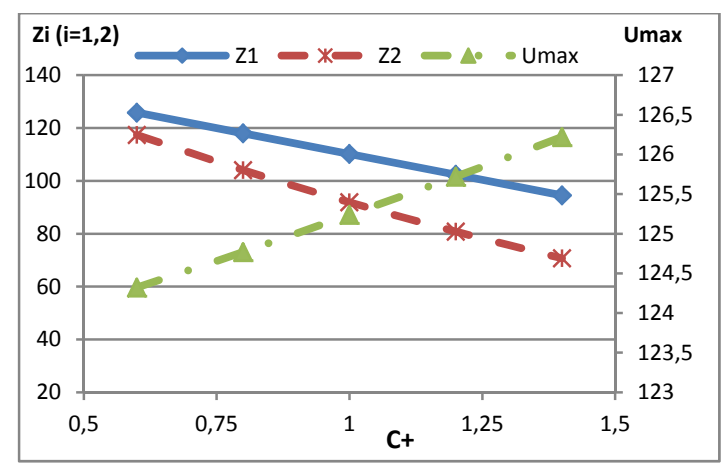

a. $\left(\mathrm{C}^{+}\right)$

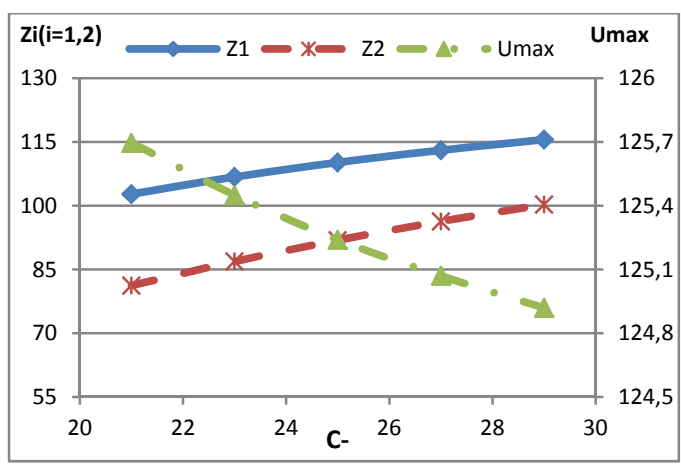

b. $\left(C^{-}\right)$

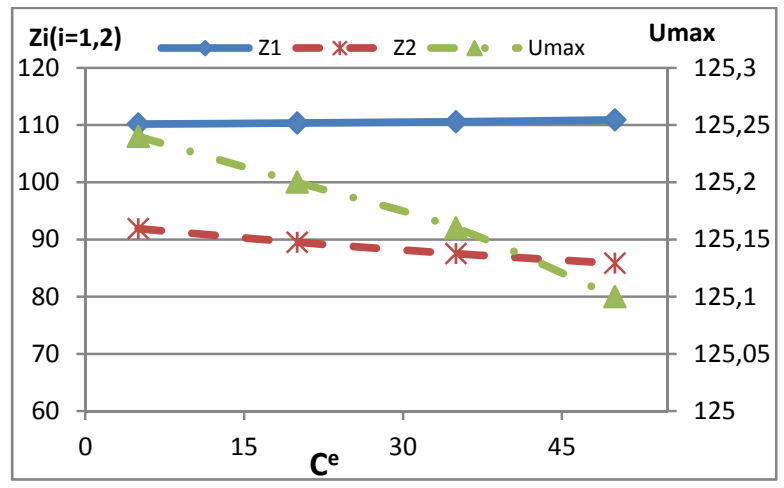

c. $\left(C^{e}\right)$

Figure 7: Variation of $\mathrm{Zi}^{*}(\mathrm{i}=1,2)$ and $\mathrm{U}_{\max }{ }^{*}$ when varying $\mathrm{C}^{+}, \mathrm{C}^{-}, \mathrm{C}^{\mathrm{e}}(\mathrm{EHPPU})$.

\section{Numerical example: Nonlinear emission rate case study}

In the previous sections, we considered a linear relationship between the emission and the production rates. This assumption could be unrealistic in some practical situations. Consequently, the exploration of other modes of emissions seems appropriate in order to study the robustness of the proposed policies.

In the literature, Jaber et al. (2013) adopted a quadratic expression given by Bogaschewsky (1995) to describe the relationship between the emission rate and the production rate.

In our case, we propose a more general expression which gives more flexibility to the emission model. This expression is given by equation (14): 


$$
\dot{e}(t)=\theta \times\left[u(t)+e^{k(u(t)-d)}\right]
$$

Where $\mathrm{k}$ is a given parameter allowing the adjustment of the shape of the relationship. Note that $\mathrm{k}$ and $\theta$ are two parameters characterizing the manufacturing system and can be found based on its history. Figure 8 illustrates the emission rate as a function of the production rate under equation (14) for different values of $\mathrm{k}$ and a fixed value of $\theta=1.1$.

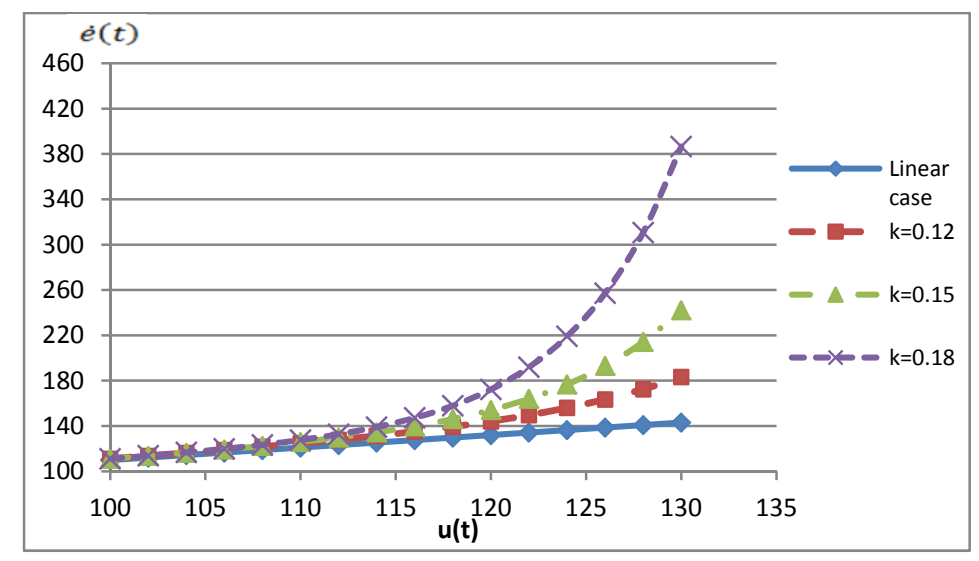

Figure 8: Emission rate evolution function of the production rate.

Using the exponential form leads to study the case where the emission rate increases in a more pronounced way as the production rate increases. We use the same simulation model, and introduce the new relationship between $\dot{e}(t)$ and $u(t)$. For $\mathrm{k}=0.15$ and $\theta=1.1$, the results show that the EHPP is better than the classical HPP in terms of incurred total cost. The average value of the gain remains around $4 \%$ as in the case of a linear emission rate.

Recall that the optimization of the production rate, in the case of a linear relationship between $\dot{e}(t)$ and $u(t)$, accentuates the gain in EHPPU and HPPU as compared to EHPP and HPP. When the relationship between $u(t)$ and $\dot{e}(t)$ is exponential, our experimental results show that the economic gain provided by the optimization of $U_{\max }$ is much more significant compared to the case of a linear emission rate. Indeed, for $\mathrm{k}=0.15$, the improvements in HPPU and EHPPU compared to HPP and EHPP are more than $22 \%$ and $29 \%$, respectively. This result is clearly logical since the penalization of the production rate by the penalty cost $\left(\mathrm{C}^{\mathrm{e}}\right)$ is very strong in the case of an exponential relationship. 


\subsection{Effect of the trajectory of the emission rate}

The parameter $\mathrm{k}$ in equation (14) measures the intensity of the relationship between $\dot{e}(t)$ and $u(t)$. At this point, it is interesting to note that the effect of the variation of $\mathrm{k}$ on the variables defining the control policies is the same as that of the cost parameter $C^{e}$. This is explained by the fact that the variation of these two parameters $\left(\mathrm{k}\right.$ and $\left.\mathrm{C}^{\mathrm{e}}\right)$ has the same consequences on the total emission cost. Indeed, if $\mathrm{k}$ increases, the emission quantity increases, and consequently, the total emission cost increases. Similarly, increasing $\mathrm{C}^{\mathrm{e}}$ also leads to an increase of the total emission cost.

From Table 7, it is important to note that the gain of HPPU and EHPPU compared to HPP and EHPP, respectively, is accentuated when $\mathrm{k}$ increases. In fact, with higher emission quantities, the production rate is greatly penalized. Therefore, the optimization of $u(t)$ becomes more profitable.

The key observation in this section is that the new policy, EHPPU, is the best policy and shows its advantage compared to the classical HPP with an economic gain of more than $46 \%$ in some cases $(\mathrm{k}=0.18)$.

Table 7: Effect of the variation of $\mathrm{k}$ on the total costs of policies

\begin{tabular}{|c|c|c|c|c|c|c|}
\cline { 2 - 7 } \multicolumn{1}{c|}{} & HPP & \multicolumn{2}{c|}{ HPPU } & EHPP & \multicolumn{2}{c|}{ EHPPU } \\
\hline $\mathbf{K}$ & Cost $^{*}$ & Cost $^{*}$ & Gain/HPP & Cost $^{*}$ & Cost $^{*}$ & Gain/HPP \\
\hline $\mathbf{0 . 1 2}$ & 157.66 & 129.74 & $17.71 \%$ & 150.45 & 116.71 & $25.97 \%$ \\
\hline $\mathbf{0 . 1 5}$ & 210.08 & 163.06 & $22.38 \%$ & 202.03 & 142.28 & $32.27 \%$ \\
\hline $\mathbf{0 . 1 8}$ & 339.01 & 197.37 & $41.78 \%$ & 329.32 & 179.72 & $46.98 \%$ \\
\hline
\end{tabular}

\subsection{Influence of cost parameters on the control policies}

In this section, a comprehensive study of the influence of cost parameters on the total incurred cost of the control policies is performed. The main objective is to identify and analyze the evolution of these policies compared to one another in the case of an exponential emission rate.

From Figure 9a (respectively Figure 9b), when $\mathrm{C}^{+}$decreases (respectively C- increases), the difference between the policies (HPP, HPPU) and between (EHPP, EHPPU) increases because the production threshold $\mathrm{Zi}^{*}(\mathrm{i}=1,2)$ increase. Thus, the system produces more at 
$\mathrm{U}_{\max }$ for (HPP, EHPP) and more at $\mathrm{U}_{\max } *\left(\leq \mathrm{U}_{\max }\right)$ in the case of (HPPU, EHPPU). As a result, the optimization of the production rate becomes more interesting. An opposite effect is observed when increasing $\mathrm{C}^{+}$(respectively $\mathrm{C}^{-}$decreases).

From Figure $9 \mathrm{c}$, when increasing $\mathrm{C}^{\mathrm{e}}$, the advantage of the production rate optimization increases, leading to a higher difference in total cost between (HPP, HPPU) and between (EHPP, EHPPU).

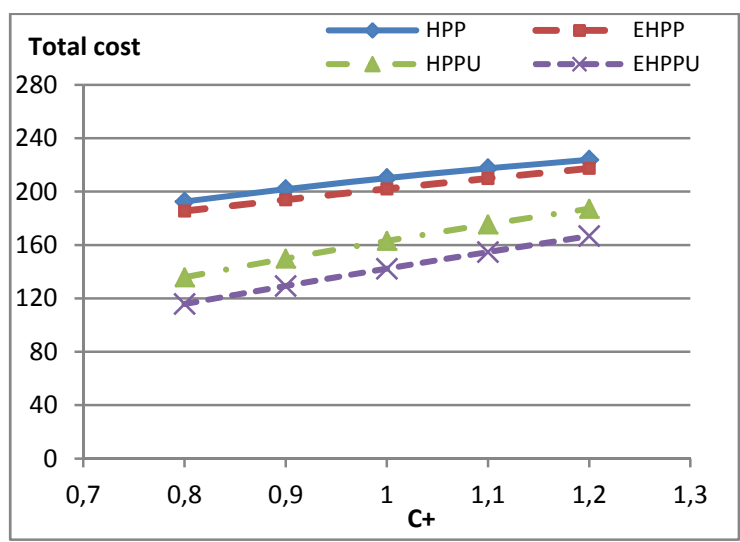

a. $\left(\mathrm{C}^{+}\right)$

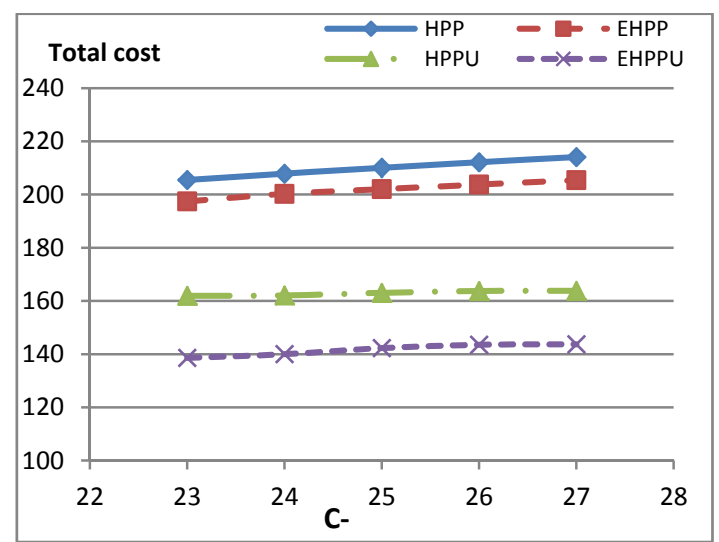

b. $\left(\mathrm{C}^{-}\right)$

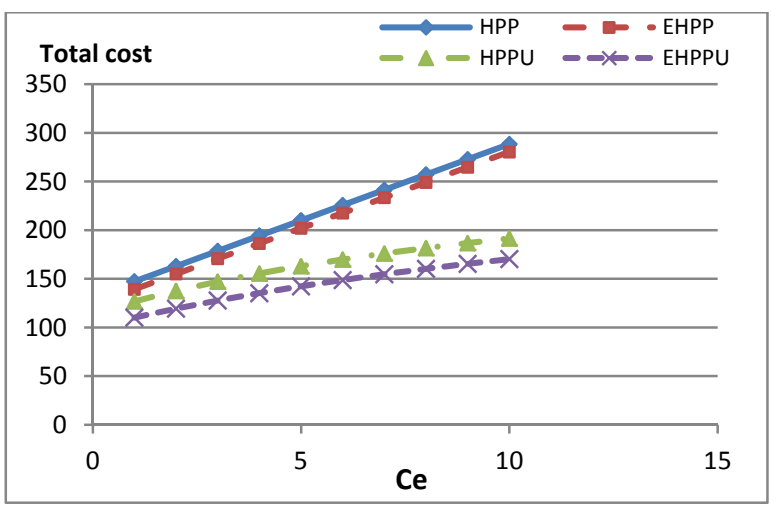

c. $\left(C^{\mathrm{e}}\right)$

Figure 9: Variation of total costs of policies

Note that the same results were observed in the case of a linear emission rate, except that the gap between the costs of policies is accentuated when the emission rate is exponential. 


\section{Conclusion}

In this paper, a new control policy called the Environmental Hedging Point Policy (EHPP), characterized by two hedging levels and the voluntary emission limit, which integrates environmental concerns in the production rate control of unreliable manufacturing systems, is proposed. The results obtained show that EHPP is more advantageous in terms of incurred costs compared to the classical HPP, with an average gain of around $4 \%$. To ensure the effectiveness of the proposal, several sensitivity analyses are conducted. To enhance the obtained gain, an improved version of EHPP, called EHPPU, which optimizes the maximum production rate, is proposed. As expected, this control policy improved the incurred total cost compared to EHPP, with an average gain of around $20 \%$. The gain of EHPPU reaches $46 \%$ compared to HPP when the emission rate evolves exponentially with respect to the production rate.

In conclusion, in a context where the relevant authorities are becoming increasingly strict about manufacturing facility operations generating harmful emissions to the environment, the proposed policies give managers valuable feedback adaptive strategies for a better control of the production rate and the emissions generated, as well as a better cost compromise.

Considered among the first works treating this type of problem in a complex environment of manufacturing systems, this work will have a significant impact on future studies in this context. Indeed, several aspects can be addressed in the next work by integrating maintenance, quality, etc.

\section{References}

Ayed, S., Dellagi, S., Rezg, N., 2012. Joint optimisation of maintenance and production policies considering random demand and variable production rate. International Journal of Production Research, 50 (23), 6870-6885.

Bai, S.X. and Elhafsi, M., 1997. Scheduling of an unreliable manufacturing system with non-resumable set-ups. Computers Ind. Eng., 32, 909-925.

Berthaut, F., Gharbi, A., Dhouib, K., 2011. Joint modified block replacement and production/inventory control policy for a failure-prone manufacturing cell. Omega, 39, 642-654.

Bonney, M. Jaber, M.J., 2011. Environmentally responsible inventory models: Nonclassical models for a non-classical era. International Journal of Production Economics, 133 (1), 43-53. 
Bogaschewsky, R., 1995. Natürliche Umwelt und Produktion. Wiesbaden: GablerVerlag.

Bouslah, B., Gharbi, A., Pellerin, R., 2013. Joint optimal lot sizing and production control policy in an unreliable and imperfect manufacturing system. Int. J. Production Economics, 144, 143-156.

Brandenburg, M., Govindan, K., Sarkis, J., \& Seuring, S., 2014. Quantitative models for sustainable supply chain management: Developments and directions. European Journal of Operational Research, 233(2), 299-312.

Brouhle, K., Griffiths, C., Wolverton, A., 2009. Evaluating the role of EPA policy levers: An examination of a voluntary program and regulatory threat in the metal-finishing industry. Journal of Environmental Economics and Management, 57, 166-181.

Chen, C. and Monahan, G.E., 2010. Environmental safety stock: The impacts of regulatory and voluntary control policies on production planning, inventory control, and environmental performance. European Journal of Operational Research, 207, $1280-1292$.

Gharbi, A., Kenne, J.P., Hajji, A., 2006. Operational level-based policies in production rate control of unreliable manufacturing systems with setups. International Journal of Production Research, 44 (3), 545-567.

Giri, B. and Dohi, T., 2005. Computational aspects of an extended EMQ model with variable production rate. Computers \& Operations Research, 32(12), 3143-3161.

Hajji, A., Gharbi, A., Kenne, J.P., 2009. Joint Replenishment and Manufacturing Activities Control in a Two Stage Unreliable Supply Chain. International Journal of Production Research, 47 (12), 3231-3251.

Hajji, A., Gharbi, A., Kenne, J.P., Pellerin, R., 2011. Production control and replenishment strategy with multiple suppliers. European Journal of Operational Research, 208, 67-74

Jaber, M.Y., Glock C.H., El Saadany A.M.A., 2013. Supply chain coordination with emissions reduction incentives. International Journal of Production Research, 51 (1), 69-82.

Kenne, J. P., \& Gharbi, A., 2000. Production planning problem in manufacturing systems with general failure and repair time distributions. Production Planning \& Control, 11(6), 581-588.

Lavoie, P., Gharbi, A. and Kenne, J.-P., 2010. A comparative study of pull control mechanisms for unreliable homogenous transfer lines. International Journal of Production Economics, 124 (1), 241-251.

Lee W.L. and Yik, F.W.H., 2004. Regulatory and voluntary approaches for enhancing building energy efficiency. Progress in Energy and Combustion Science, 30, 477-499.

Marcus, P.A. and Willig, J.T., 1997. Moving Ahead with ISO 14000: Improving Environmental Management and Advancing Sustainable Development. John Wiley \& Sons, New York.

Montgomery, D.C., 2005. Design and analysis of experiments. New York: John Wiley \& Sons.

Porter, M.E. and Van der Linde, C., 1995. Green and competitive: Ending the stalemate. Harvard Business Review, 73 (5), 120-134. 
Radhoui, M., Rezg, N., Chelbi, A., 2009. Integrated model of preventive maintenance, quality control and buffer sizing for unreliable and imperfect production systems, International Journal of Production Research, 47 (2), 389-402.

Rezg N, Chelbi A, Xie X., 2005. Modeling and optimizing a joint inventory control and preventive maintenance strategy for a randomly failing production unit: analytical and simulation approaches. International Journal of Computer Integrated Manufacturing, 18(2-3), 225-35.

Sana, S. S. and Chaudhuri, K., 2010. An EMQ model in an imperfect production process. International Journal of Systems Science, 41(6), 635-646.

Simonet, E., 2003. Les Systèmes de Management Environnemental - Synthèse. Association LaPlateforme. Web site: www.Laplateforme.org. Date of consultation: 20-02-2014. 